\title{
ON NONINNER AUTOMORPHISMS OF FINITE NONABELIAN $p$-GROUPS
}

\author{
S. M. GHORAISHI
}

(Received 26 January 2013; accepted 20 March 2013; first published online 7 June 2013)

\begin{abstract}
A long-standing conjecture asserts that every finite nonabelian $p$-group has a noninner automorphism of order $p$. In this paper the verification of the conjecture is reduced to the case of $p$-groups $G$ satisfying $Z_{2}^{\star}(G) \leq C_{G}\left(Z_{2}^{\star}(G)\right)=\Phi(G)$, where $Z_{2}^{\star}(G)$ is the preimage of $\Omega_{1}\left(Z_{2}(G) / Z(G)\right)$ in $G$. This improves Deaconescu and Silberberg's reduction of the conjecture: if $C_{G}(Z(\Phi(G))) \neq \Phi(G)$, then $G$ has a noninner automorphism of order $p$ leaving the Frattini subgroup of $G$ elementwise fixed ['Noninner automorphisms of order $p$ of finite $p$-groups', J. Algebra 250 (2002), 283-287].
\end{abstract}

2010 Mathematics subject classification: primary 20D15; secondary 20D45.

Keywords and phrases: finite $p$-groups, automorphisms, noninner automorphisms.

\section{Introduction}

Let $p$ be a prime and $G$ be a finite nonabelian $p$-group. A longstanding conjecture asserts that $G$ has a noninner automorphism of order $p$ [12, Problem 4.13]. This conjecture is still open. In fact, the statement of the conjecture is a sharpened version of a well-known and nontrivial property of finite $p$-groups that, with the exception of groups of order $p$, they always have a noninner automorphism of $p$-power order [7].

The conjecture has been established for $p$-groups of class 2 and $3[2,3,11]$, for regular $p$-groups [13], for $p$-groups $G$ in which $G / Z(G)$ is powerful [1], for $p$-groups $G$ in which $(G, Z(G))$ is a Camina pair and $p \neq 2$ [9], for 2-groups with a cyclic commutator subgroup [10], and for $p$-groups of order $p^{m}$ and exponent $p^{m-2}$ [14]. It is worth noting that most of the noninner automorphisms given in these results leave either $\Phi(G)$ or $Z(G)$ elementwise fixed. Also, Deaconescu and Silberberg have proved that if $C_{G}(Z(\Phi(G))) \neq \Phi(G)$, then $G$ has a noninner automorphism of order $p$ leaving $\Phi(G)$ elementwise fixed [5]. Hence, they have reduced the verification of the conjecture to the degenerate case in which

$$
C_{G}(Z(\Phi(G)))=\Phi(G)
$$

The author gratefully acknowledges the moral and financial support of the University of Isfahan as well as its Centre of Excellence for Mathematics. This research was in part supported by a grant from IPM (No. 91050219).

(C) 2013 Australian Mathematical Publishing Association Inc. 0004-9727/2013 \$16.00 
The main motivation of the present paper is to reduce the verification of the conjecture further. In addition, our aim is to find a noninner automorphism of order $p$ which acts trivially on a maximal subgroup of $G$. Let $Z_{2}^{\star}(G) / Z(G)=\Omega_{1}\left(Z_{2}(G) / Z(G)\right)$, where for a finite $p$-group $H, \Omega_{1}(H)=\left\langle h \in H \mid h^{p}=1\right\rangle$. Our main results are as follows.

THEOREM 1.1. Let $p$ be a prime and $G$ be a finite nonabelian p-group. If $G$ fails to fulfil the condition

$$
Z_{2}^{\star}(G) \leq C_{G}\left(Z_{2}^{\star}(G)\right)=\Phi(G),
$$

then $G$ has a noninner automorphism of order p leaving the Frattini subgroup of $G$ elementwise fixed. Moreover, if $p$ is odd, then the noninner automorphism can be taken such that it acts trivially on a maximal subgroup of $G$.

Theorem 1.1 reduces the verification of the conjecture to the case of finite $p$-groups satisfying (**). Let $\mathscr{G}_{p}^{*}$ and $\mathscr{G}_{p}^{* *}$ denote the sets of all finite $p$-groups with the properties $(*)$ and $(* *)$, respectively. Then the following theorem holds.

Theorem 1.2. For every prime $p, \mathscr{G}_{p}^{* *} \subseteq \mathscr{G}_{p}^{*}$ and $\mathscr{G}_{p}^{*} \backslash \mathscr{G}_{p}^{* *}$ contains infinitely many p-groups.

Therefore the result of this paper extends known classes of finite $p$-groups for which the conjecture holds.

\section{Preliminaries}

Let $G$ be a finite nonabelian $p$-group. By $\mathscr{M}(G)$ we denote the set of all maximal subgroups of $G$. If $x \in G$ and $H \leq G$, then $\bar{x}$ and $\bar{H}$ denote the coset $x \Phi(G)$ and the quotient group $H \Phi(G) / \Phi(G)$, respectively. The inner automorphism of $G$ induced by $x$ is denoted by $\theta_{x}$. Also, we denote the direct product of groups $G_{1}, G_{2}, \ldots, G_{n}$, by $\operatorname{Dr}_{i=1}^{n} G_{i}$. Any unexplained notation is standard and follows that of [8]. We use the following facts in the proofs.

Remark 2.1. Let $n \in \mathbb{N}, x, y \in G$ and $a \in Z_{2}(G)$.

$$
\begin{array}{ll}
\text { - } & (x a)^{n}=x^{n} a^{n}[a, x]^{\left(\begin{array}{c}
n \\
2
\end{array}\right) .} \\
\text { - } & {\left[x^{n}, a\right]=[x, a]^{n}=\left[x, a^{n}\right] .} \\
\text { - } & {[x, a y]=[x, a][x, y] .} \\
\text { - } & \text { Moreover, if } a^{p} \in Z(G) \text { then }[a, \Phi(G)]=1 .
\end{array}
$$

Remark 2.2. Let $G$ be a finite $p$-group, $M$ be a maximal subgroup of $G$ and $g \in G \backslash M$. Let $u \in Z(M)$ such that $(g u)^{p}=g^{p}$. Then the map $\alpha$ given by $g \mapsto g u$ and $m \mapsto m$, for all $m \in M$, can be extended to an automorphism of order $|u|$ that acts trivially on $M$.

Remark 2.3 [5, Remark 4]. Let $G$ be a central product of subgroups $A$ and $B$; that is, $G=A B$ and $[A, B]=1$. Suppose that $\alpha \in \operatorname{Aut}(A)$ and $\beta \in \operatorname{Aut}(B)$ agree on $A \cap B$. Then $\alpha$ and $\beta$ admit a common extension $\gamma \in \operatorname{Aut}(G)$. In particular, if $A$ has a noninner automorphism of order $p$ which fixes $Z(A)$, then $G$ has a noninner automorphism of order $p$ which fixes $Z(A)$ and $B$. 
Remark 2.4. Let $A$ and $B$ be two elementary abelian finite $p$-groups. The set of all homomorphisms from $A$ to $B$, which is denoted by $\operatorname{Hom}(A, B)$, forms an elementary abelian $p$-group by + operation (that is, $(f+g)(a)=f(a) g(a)$ for $f, g \in \operatorname{Hom}(A, B)$ and $a \in A)$. Let $A=\operatorname{Dr}_{i=1}^{m}\left\langle a_{i}\right\rangle$ and $B=\operatorname{Dr}_{i=1}^{n}\left\langle b_{i}\right\rangle$, where $m=d(A)$ and $n=d(B)$. For $1 \leq i \leq m$ and $1 \leq j \leq n$, the map $f_{i, j}: A \rightarrow B$ defined by $a_{k} \mapsto b_{j}^{\delta_{k, i}}$, where $\delta$ is the Kronecker delta, can be extended to a homomorphism from $A$ to $B$. Furthermore $\left\{f_{i, j} \mid 1 \leq i \leq m, 1 \leq j \leq n\right\}$ is a minimal generating set for $\operatorname{Hom}(A, B)$. Thus $\operatorname{Hom}(A, B) \cong \operatorname{Dr}_{i=1}^{n} A$ is of $\operatorname{rank} d(A) d(B)$.

The latter remark becomes obvious when it is realised that $A$ and $B$ are vector spaces over the field of $p$ elements.

Remark 2.5. Let $G$ be a finite nonabelian $p$-group such that $\Omega_{1}(Z(G)) \leq \Phi(G)$. If $f \in \operatorname{Hom}\left(\bar{G}, \Omega_{1}(Z(G))\right)$ then the map $\sigma_{f}: G \rightarrow G$ defined by $x \mapsto x f(\bar{x})$ is an automorphism of order $p$. In addition, if $\operatorname{ker}(f) \in \mathscr{M}(\bar{G})$ then $\sigma_{f}$ acts trivially on a maximal subgroup of $G$.

\section{Proofs of the main results}

Let $Z_{2}^{\star}(G) / Z(G)=\Omega_{1}\left(Z_{2}(G) / Z(G)\right)$. In the following lemmas we derive some properties of $Z_{2}^{\star}(G)$.

LeMma 3.1. If $G$ is a finite p-group, then $\left[Z_{2}^{\star}(G), \Phi(G)\right]=1$.

Proof. This follows immediately from Remark 2.1 .

Lemma 3.2. Let $H \leq G$ and $a \in Z_{2}^{\star}(G)$. Then the $\operatorname{map}_{H} \varphi_{a}: \bar{H} \rightarrow \Omega_{1}(Z(G))$, given by $\bar{h} \mapsto[h, a]$, for $h \in H$, is a homomorphism. Also, the map

$$
{ }_{H} \varphi: Z_{2}^{\star}(G) \longrightarrow \operatorname{Hom}\left(\bar{H}, \Omega_{1}(Z(G)),\right.
$$

defined by $a \mapsto{ }_{H} \varphi_{a}$, for $a \in Z_{2}^{\star}(G)$, is a homomorphism and $\operatorname{ker}\left({ }_{H} \varphi\right)=Z_{2}^{\star}(G) \cap C_{G}(H)$.

Proof. This is straightforward.

The following propositions relate $Z_{2}^{\star}(G)$ to the automorphisms of order $p$ which act trivially on a maximal subgroup of $G$.

Proposition 3.3. Let $p$ be an odd prime and $G$ be a finite nonabelian p-group such that $Z(G)$ is cyclic and $Z_{2}^{\star}(G) / Z(G)$ is not cyclic. Then $G$ has a noncentral automorphism of order p leaving a maximal subgroup of $G$ elementwise fixed.

Proof. By hypothesis, $Z_{2}^{\star}(G) / Z(G)=\langle a Z(G)\rangle \times\langle b Z(G)\rangle \times L / Z(G)$, for some $a, b \in$ $Z_{2}^{\star}(G) \backslash Z(G)$ and $L \leq Z_{2}^{\star}(G)$. Since $Z(G)$ is cyclic, we may assume that $b^{p}=a^{p^{i} j}$, for some integers $i, j$. Let $u=b a^{p^{i-1} j}, M=C_{G}(u)$ and ${ }_{G} \varphi_{u}$ be the homomorphism given in Lemma 3.2. Then it follows that $M=\operatorname{ker}\left({ }_{G} \varphi_{u}\right) \in \mathscr{M}(G)$. Now let $g \in G \backslash M$. Since $u$ is an element of order $p$ in $Z_{2}^{\star}(G) \backslash Z(G)$ and $(g u)^{p}=g^{p}$, the result follows from Remark 2.2. 
Proposition 3.4. Let $G$ be a finite nonabelian p-group and $H \leq G$. If

$$
d\left(Z_{2}^{\star}(G) / Z_{2}^{\star}(G) \cap C_{G}(H)\right) \neq d(\bar{H}) d(Z(G)),
$$

then $G$ has a central noninner automorphism of order $p$ which acts trivially on a maximal subgroup of $G$.

Proof. By a well-known argument (or applying Remark 2.2), we may assume that $Z(G) \leq \Phi(G)$. Now, let ${ }_{H} \varphi$ be the homomorphism given in Lemma 3.2. Then

$$
\operatorname{ker}\left({ }_{H} \varphi\right)=\frac{Z_{2}^{\star}(G)}{Z_{2}^{\star}(G) \cap C_{G}(H)}
$$

By hypothesis, $H \not \leq \Phi(G)$ and ${ }_{H} \varphi$ is not an epimorphism. Thus for some $1 \leq i \leq d(\bar{H})$ and $1 \leq j \leq d(Z(G)), f_{i, j} \notin \operatorname{Im}(\varphi)$, where $f_{i, j}$ is as in Remark 2.4. If necessary, extend $\left\{x_{1}, \ldots, x_{s}\right\}$ to a minimal generating set $\left\{x_{1}, \ldots, x_{s}, \ldots, x_{d}\right\}$ of $G$. For $1 \leq k \leq d$, set

$$
f\left(\overline{x_{k}}\right)= \begin{cases}f_{i, j}\left(\overline{x_{k}}\right) & 1 \leq k \leq s \\ 1 & s<k \leq d\end{cases}
$$

Then $f$ determines an element of $\operatorname{Hom}\left(\bar{G}, \Omega_{1}(Z(G))\right)$. By Remark 2.5, $\sigma_{f}$ is an automorphism of $G$ of order $p$ that fixes a maximal subgroup of $G$ elementwise. If $\sigma_{f}=\theta_{a}$ is inner, then one must have $a \in Z_{2}^{\star}(G)$. Thus for $x \in H, \sigma_{f}(x)=\theta_{a}(x)$ and hence

$$
f_{i, j}(\bar{x})=x^{-1} \sigma_{f}(x)=[x, a]=\varphi_{a}(\bar{x}) .
$$

This means that $f_{i, j} \in \operatorname{Im}(\varphi)$, a contradiction. Therefore $\sigma_{f}$ is noninner and the result follows.

Proposition 3.5. Let $p$ be a prime and $G$ be a finite p-group. If $C_{G}\left(Z_{2}^{\star}(G)\right) \neq \Phi(G)$, then $G$ has a central noninner automorphism of order p leaving a maximal subgroup of $G$ elementwise fixed.

Proof. Assume that $G$ is a counterexample to the theorem. Let $M \in \mathscr{M}(G)$ and $g \in G \backslash M$. Let $u$ be an element of order $p$ in $Z(G) \cap M$. Then by Remark 2.2 the map $\alpha$ given by $g \mapsto g u$ and $m \mapsto m$, for all $m \in M$, can be extended to an automorphism of order $p$ that leaves $M$ elementwise fixed. By assumption $\alpha=\theta_{x_{M}}$, for some $x_{M} \in G$. Therefore $x_{M} \in Z_{2}^{\star}(G)$ and $M=C_{G}\left(x_{M}\right)$. By Lemma 3.1, $\Phi(G) \leq C_{G}\left(Z_{2}^{\star}(G)\right)$. Therefore

$$
\Phi(G) \leq C_{G}\left(Z_{2}^{\star}(G)\right) \leq \bigcap_{M \in \mathscr{M}(G)} C_{G}\left(x_{M}\right)=\bigcap_{M \in \mathscr{M}(G)} M=\Phi(G),
$$

and the result follows. 
Second Proof. Let $G$ be a counterexample to the theorem. For $x \in C_{G}\left(Z_{2}^{\star}(G)\right)$, let $H=\langle\bar{x}\rangle$. Then it follows from Proposition 3.4, that $x \in \Phi(G)$. Therefore $C_{G}\left(Z_{2}^{\star}(G)\right) \leq$ $\Phi(G)$. Now the result follows from Lemma 3.1.

Proposition 3.6. Let $p$ be a prime and $G$ be a finite p-group of class 2 . If either $p>2$ or $Z(G)$ is not cyclic then $\operatorname{Aut}(G)$ contains a noninner automorphism of order p leaving a maximal subgroup of $G$ elementwise fixed. In addition, if $Z(G)$ is not cyclic, then the noninner automorphism can be taken to be central.

Proof. Let $G$ be a counterexample to the proposition. By Theorem 3.5, $C_{G}\left(Z_{2}^{\star}(G)\right)=$ $\Phi(G)$. Thus $Z(G) \leq \Phi(G)$ and since $G$ is of class 2 , one has $d\left(Z_{2}^{\star}(G) / Z(G)\right)=$ $d(G / Z(G))=d(G)$. Now if $d(Z(G))>1$, then the result follows from Proposition 3.4, and if $d(Z(G))=1$ and $p>2$, then Proposition 3.3 completes the proof.

Theorem 3.6 does not hold for 2-groups of class 2 in general. Indeed, there are examples of groups of class 2 in which every automorphism of order two fixing $\Phi(G)$ elementwise is inner $[1,11]$.

The following result improves [11, Part (1) of Theorem].

Proposition 3.7. Let $p$ be a prime and $G$ be a finite p-group such that $Z_{2}^{\star}(G)$ is not abelian. If $p$ is odd then $G$ has a noninner automorphism of order p leaving a maximal subgroup of $G$ elementwise fixed, and if $p=2$ then $G$ has a noninner automorphism of order two leaving the Frattini subgroup of $G$ elementwise fixed.

The proof of Proposition 3.7 requires the following preliminary fact. Recall that a finite nonabelian $p$-group, all of whose maximal subgroups are abelian, is called a minimal nonabelian $p$-group or Rédei $p$-group.

Remark 3.8. Let $G$ be a Rédei $p$-group. If $p$ is odd then $G$ has a noninner automorphism of order $p$ leaving a maximal subgroup of $G$ elementwise fixed, and if $p=2$ then $G$ has a noninner automorphism of order $p$ leaving $\Phi(G)$ elementwise fixed. The former follows from Theorem 3.6, since Rédei $p$-groups have nilpotency class 2 , and the latter has been proved by using the classification of Rédei 2-groups [5, Remark 3].

Proof of Proposition 3.7. Assume that $G$ is a counterexample of minimal order to the proposition.

First we prove that $\overline{Z_{2}^{\star}(G)}$ is not cyclic. Suppose to the contrary that $\overline{Z_{2}^{\star}(G)}=\langle\bar{u}\rangle$, for some $u \in Z_{2}^{\star}(G)$. If $x, y \in Z_{2}^{\star}(G)$, then $x=u^{i} a$ and $y=u^{j} b$ for some $i, j \in \mathbb{N}$ and $a, b \in \Phi(G) \cap Z_{2}^{\star}(G)$. Now it follows from Lemma 3.1 that $[x, y]=1$. But this means that $Z_{2}^{\star}(G)$ is abelian, a contradiction.

Then, by Proposition 3.4,

$$
d\left(Z_{2}^{\star}(G) / Z_{2}^{\star}(G) \cap C_{G}\left(Z_{2}^{\star}(G)\right)\right)=d\left(\overline{Z_{2}^{\star}(G)}\right) d(Z(G)) ;
$$

and by Proposition 3.5, $C_{G}\left(Z_{2}^{\star}(G)\right)=\Phi(G)$. Therefore $Z(G)$ is cyclic. 
Next, suppose that $a, b \in Z_{2}^{\star}(G)$ such that $[a, b] \neq 1$. Let $K=\langle a, b\rangle$ and $L=C_{G}(K)$. Note that $[K, G]=K^{\prime}=\Omega_{1}(Z(G))=\langle[a, b]\rangle$. Hence, if $x \in G$, then $[a, x]=[a, b]^{s}$ and $[b, x]=[a, b]^{t}$, for some integers $s, t$. Thus, $\left[a, b^{-s} a^{t} x\right]=1$ and $\left[b, b^{-s} a^{t} x\right]=1$. Therefore $b^{-s} a^{t} x \in C_{G}(K)$ and it follows that $G$ is the central product of $K$ and $L$. Moreover, $K$ is a Rédei $p$-group. Hence, by Remark 3.8, $K \supsetneqq G$.

Finally, if $p$ is odd, then by assumption $K$ has a noninner automorphism $\alpha$ of order $p$ that acts trivially on a maximal subgroup $M$ of $K$. By Remark 2.3, $\alpha$ can be extended to a noninner automorphism of $G$ of order $p$ that fixes $M L$. Since $Z(K)=\left\langle[a, b], a^{p}, b^{p}\right\rangle=\Phi(K)$, we have $K \cap L=Z(K)=\Phi(K)=M \cap L$ and

$$
\frac{|G|}{|M L|}=\frac{|K||L| /|K \cap L|}{|M||L| /|M \cap L|}=\frac{|K|}{|M|}=p .
$$

Therefore $M L \in \mathscr{M}(G)$, a contradiction. Also, if $p=2$, then a similar argument gives a contradiction.

Proof of Theorem 1.1. This follows immediately form Propositions 3.5 and 3.7.

To prove Theorem 1.2, we use the following observation.

Lemma 3.9. If $G_{1}$ belongs to $\mathscr{G}_{p}^{*} \backslash \mathscr{G}_{p}^{* *}$, then so does $G_{1} \times G_{2}$, for all $G_{2} \in \mathscr{G}_{p}^{*}$.

Proof. The result follows immediately from the following elementary facts. Let $G_{1}$ and $G_{2}$ be two finite $p$-groups. Let $H_{1} \leq G_{1}$ and $H_{2} \leq G_{2}$. Set $G=G_{1} \times G_{2}$ and $H=H_{1} \times H_{2}$. Then $\Phi(G)=\Phi\left(G_{1}\right) \times \Phi\left(G_{2}\right)$ and $C_{G}(H)=C_{G_{1}}\left(H_{1}\right) \times C_{G_{2}}\left(H_{2}\right)$.

Proof of Theorem 1.2. Let $G \in \mathscr{G}_{p}^{* *}$. Then by Lemma 3.1, $Z_{2}^{\star}(G) \leq Z(\Phi(G))$. Therefore,

$$
\Phi(G)=C_{G}\left(Z_{2}^{\star}(G)\right) \geq C_{G}(Z(\Phi(G))) \geq \Phi(G) .
$$

This proves the first part of the theorem. For the second part, by Lemma 3.9 it suffices to show that for every prime $p, \mathscr{G}_{p}^{*} \backslash \mathscr{G}_{p}^{* *} \neq \varnothing$. First, assume that $p>3$ and let $G$ be a group with the following power-commutator presentation:

$$
\begin{aligned}
& G=\operatorname{Pc}\left\langle g_{1}, g_{2}, g_{3}, g_{4}, g_{5}\right| g_{1}^{p}=g_{2}^{p}=g_{3}^{p}=g_{4}^{p}=g_{5}^{p}=1, \\
& \quad g_{3}=\left[g_{2}, g_{1}\right], g_{4}=\left[g_{3}, g_{1}\right], g_{5}=\left[g_{4}, g_{1}\right], \\
& {\left[g_{5}, g_{1}\right]=1,\left[g_{3}, g_{2}\right]=g_{5},\left[g_{4}, g_{2}\right]=1,\left[g_{5}, g_{2}\right]=1} \\
& \left.\left[g_{4}, g_{3}\right]=1,\left[g_{5}, g_{3}\right]=1,\left[g_{5}, g_{4}\right]=1\right\rangle,
\end{aligned}
$$

To show the consistency of this presentation, it suffices to check that for each of the following pairs of test words the collections of both words coincide (see [15, page 424] and [4, Lemma 2.1]).

(i) $\left(g_{k} g_{j}\right) g_{i}$ and $g_{k}\left(g_{j} g_{i}\right)$, for $1 \leq i<j<k \leq 5$,

(ii) $g_{i}$ and $g_{j}^{p-1}\left(g_{j} g_{i}\right)$, for $1 \leq i<j \leq 5$,

(iii) $g_{j}$ and $\left(g_{j} g_{i}\right) g_{i}^{p-1}$, for $1 \leq i<j \leq 5$. 
Checking (i) is straightforward and one may use induction to check (ii) and (iii). For instance, by induction on $i$, we get $g_{2} g_{1}^{i}=\left(g_{2} g_{1}\right) g_{1}^{i-1}=g_{1}^{i} g_{2} g_{3}^{i} g_{4}^{i(i-1) / 2} g_{5}^{i(i-1)(i-2) / 6}$. Therefore the collection of $\left(g_{2} g_{1}\right) g_{1}^{p-1}$ coincides with $g_{2}$.

Now the consistency of the presentation implies that $G$ is of order $p^{5}$ and class 4 . Thus $G$ is of maximal class. Let $Z_{2}(G) / Z(G)=\langle u Z(G)\rangle$, for some $u \in Z_{2}(G)$. Then by an easy argument as in the proof of Proposition 3.3, $C_{G}\left(Z_{2}^{\star}(G)\right)=C_{G}(u) \neq \Phi(G)$. On the other hand, $\Phi(G)=G^{\prime}$ is abelian and $C_{G}(Z(\Phi(G)))=\Phi(G)$. Therefore, $G \in \mathscr{G}_{p}^{*} \backslash \mathscr{G}_{p}^{* *} \neq \varnothing$.

Now suppose that $p \leq 3$. We use the following code in GAP [6] to complete the proof in this case.

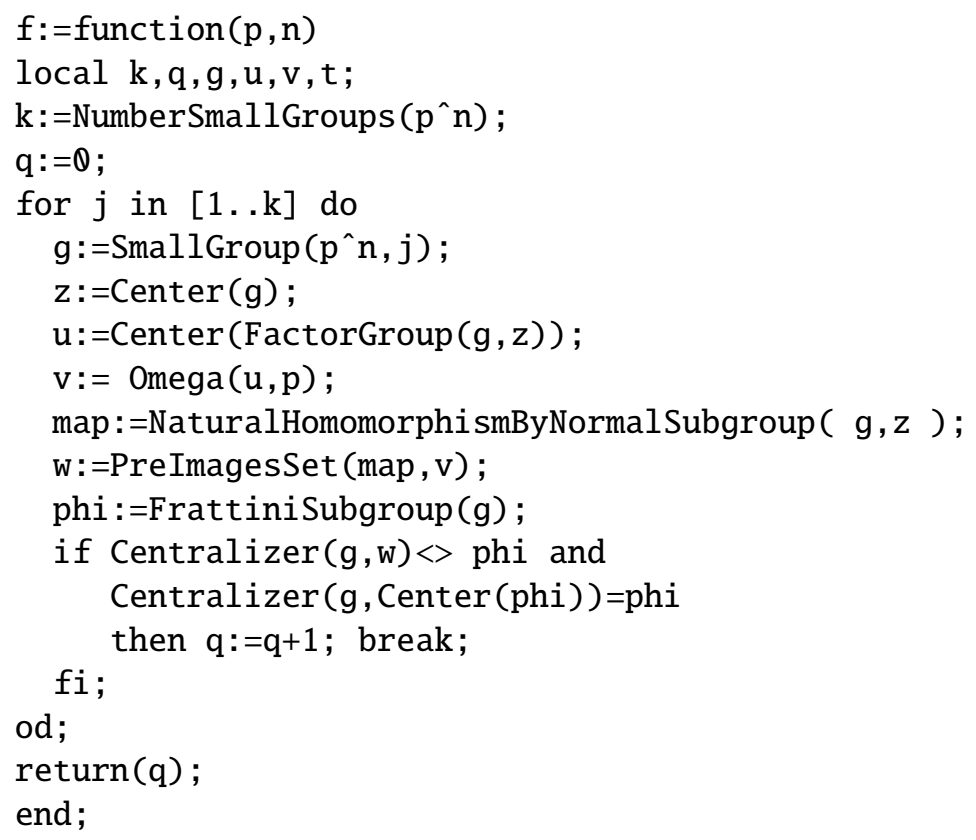

This code accepts prime $p$ and positive integer $n$. Then it returns 1 if there exists a group $G$ of order $p^{n}$ in the GAP small groups library such that $G \in \mathscr{G}_{p}^{*} \backslash \mathscr{G}_{p}^{* *}$, otherwise it returns 0 . We see that $f(2,7)=1$ and $f(3,5)=1$, which completes the proof of the theorem.

We end the paper by answering the natural question that arises here: 'Is there any finite $p$-group of class two in $\mathscr{G}_{p}^{*} \backslash \mathscr{G}_{p}^{* *}$ ?'

Proposition 3.10. Let $G$ be a finite nonabelian p-group of class 2 . Then $G \in \mathscr{G}_{p}^{*}$ if and only if $G \in \mathscr{G}_{p}^{* *}$.

Proof. By Theorem 1.2, it is enough to prove the 'only if' part. In fact we prove that if $G \in \mathscr{G}_{p}^{*}$ is of class 2 , then $Z_{2}^{\star}(G)=Z(\Phi(G))$. Suppose that $G$ is a finite $p$-group of class 2 such that $C_{G}\left(Z(\Phi(G))=\Phi(G)\right.$. Then $C_{G}(\Phi(G))=Z(\Phi(G))$ and it follows from 
Lemma 3.1 that $Z_{2}^{\star}(G) \leq Z(\Phi(G))$. Now let $a \in Z(\Phi(G))$. Thus $1=\left[a, x^{p}\right]=\left[a^{p}, x\right]$, for every $x \in G$. Therefore $a^{p} \in Z(G)$ which means that $a \in Z_{2}^{\star}(G)$. Hence $Z(\Phi(G)) \leq$ $Z_{2}^{\star}(G)$, and the result follows.

\section{Acknowledgement}

The author is grateful to the referee for valuable comments.

\section{References}

[1] A. Abdollahi, 'Powerful $p$-groups have noninner automorphisms of order $p$ and some cohomology', J. Algebra 323 (2010), 779-789.

[2] A. Abdollahi, 'Finite $p$-groups of class 2 have noninner automorphisms of order $p$ ', J. Algebra 312 (2007), 876-879.

[3] A. Abdollahi, M. Ghoraishi and B. Wilkens, 'Finite $p$-groups of class 3 have noninner automorphisms of order p', Beiträge Algebra Geom. 54(1) (2013), 363-381.

[4] K. Dekimpe and B. Eick, 'Computational aspects of group extensions and thier application in topology', Exp. Math. 11(2) (2002), 183-200.

[5] M. Deaconescu and G. Silberberg, 'Noninner automorphisms of order $p$ of finite $p$-groups', J. Algebra 250 (2002), 283-287.

[6] The GAP group, GAP-Groups, Algorithms, and Programming, version 4.4.12, www.gapsystem.org (2008).

[7] W. Gaschütz, 'Nichtabelsche $p$-Gruppen besitzen äussere p-Automorphismen', J. Algebra 4 (1966), 1-2.

[8] D. Gorenstein, Finite Groups (Harper \& Row, New York, 1968).

[9] S. M. Ghoraishi, 'A note on automorphisms of finite p-groups', Bull. Aust. Math. Soc. 87 (2013), 24-26.

[10] A. R. Jamalli and M. Viseh, 'On the existence of noinner automorphisms of order two in finite 2-groups', Bull. Aust. Math. Soc. 87 (2013), 278-287.

[11] H. Liebeck, 'Outer automorphisms in nilpotent $p$-groups of class 2', J. Lond. Math. Soc. 40 (1965), 268-275.

[12] V. D. Mazurov and E. I. Khukhro (eds), Unsolved Problems in Group Theory, The Kourovka Notebook, 16 (Russian Academy of Sciences, Siberian Division, Institue of Mathematics, Novosibirisk, 2006).

[13] P. Schmid, 'A cohomological property of regular p-groups', Math. Z. 175 (1980), 1-3.

[14] M. Shabani-Attar, 'Existence of noninner automorphisms of order $p$ in some finite $p$-groups', Bull. Aust. Math. Soc. 87 (2013), 272-277.

[15] C. C. Sims, Computation with Finitely Presented Groups (Cambridge University Press, Cambridge, 1994).

\section{S. M. GHORAISHI, Department of Mathematics, University of Isfahan, 81746-73441, Iran \\ e-mail: m.ghoraishi@sci.ui.ac.ir}

